\title{
ISLAS EN EL MAR: PARTICIPACIÓN Y DESCENTRALIZACIÓN EN “SISTEMAS INTEGRADOS" EN EDUCACIÓN
}

\author{
D. BRENT EDWARDS JR. ${ }^{1}$ \\ MaUricio Erazo ${ }^{2}$ \\ Pauline Martin ${ }^{3}$
}

\begin{abstract}
RESUMEN: Este ensayo ofrece, desde una perspectiva sistémica, hallazgos sobre el funcionamiento de la reciente estrategia de descentralización educativa en El Salvador, denominada Sistema Integrado de Escuela Inclusiva de Tiempo Pleno (SI-EITP), con el objetivo de conocer el sentido de su funcionamiento y la experiencia de la participación de la comunidad educativa inmersa en sus dinámicas. Por medio de una revisión de los documentos relacionados con el diseño y la ejecución, el análisis revela que SI-EITP no funcionó según lo esperado, debido tanto a una falta de cohesión conceptual, como a la inexistencia de un marco legal adecuado y de recursos suficientes para lograr una participación y empoderamiento locales.
\end{abstract}

Palabras-clave: Gobernanza escolar. Descentralización. Redes escolares. Participación. Educación.

\section{ISLANDS IN THE SEA: PARTICIPATION AND DESCENTRALIZATION IN 'INTEGRATED SYSTEMS' IN EDUCATION}

\begin{abstract}
This essay offers, from a systemic perspective, findings on the functioning of a recent education decentralization strategy in El Salvador, called Integrated Systems of Full-time Inclusive Schools (IS-FTIS, or SI-EITP; from Spanish, sistema integrado de escuela inclusiva de tiempo pleno), with the objective of understanding how it functions in practice and the experience of communities immersed in its dynamics. Through a compilation of documents related to its design and implementation, the analysis reveals that IS-FTIS (SI-EITP) did not work as expected, due to a lack of conceptual cohesion, as well as an inadequate legal framework, and insufficient resources to achieve local participation and empowerment.
\end{abstract}

Keywords: School governance. Decentralization. School networks. Participation. Education.

\footnotetext{
1.University of Hawaỉi at Mānoa - College of Education - Department of Educational Foundations - Honolulu (Hawaỉi), Estados Unidos. E-mail: donalde@hawaii.edu

2.Investigador independiente - Santa Tecla, El Salvador. E-mail: mauricio94erazo@gmail.com

3.Universidad Centroamericana “José Simeón Cañas" - Departamento de Ciencias de la Educación - Antiguo Cuscatlán, El Salvador. E-mail: pmartin@uca.edu.sv

Editor de sección: Adriana Dragone Silveira
} 


\title{
ILHAS NO MAR: PARTICIPAÇÃO E DESCENTRALIZAÇÃO EM “SISTEMAS INTEGRADOS” NA EDUCAÇÃO
}

\begin{abstract}
RESUMO: Este ensaio oferece, a partir de uma perspectiva sistêmica, achados sobre o funcionamento da recente estratégia de descentralização educacional em El Salvador, denominada Sistema Integrado da Escola Inclusiva em Tempo Integral (SI-EI|TP; do espanhol, sistema integrado de escuela inclusiva de tiempo pleno), com o objetivo de conhecer o sentido de seu funcionamento e sua experiência com a participação da comunidade educacional imersa em suas dinâmicas. Por meio de uma revisão dos documentos relacionados com o projeto e a execução, a análise revela que o SI-EITP não funcionou como esperado, em virtude tanto de uma falta de coesão conceitual quanto da ausência de um marco legal adequado e de recursos suficientes para alcançar uma participação e um empoderamento locais.
\end{abstract}

Palavras-chave: Governança escolar. Descentralização. Redes escolares. Participação. Educação.

\section{Introducción}

$\mathrm{E}$ n América Latina, a pesar de que se utilizaron mejores insumos y se realizaron cambios en la gestión que contribuyeron a expandir el acceso a los sistemas escolares durante la década de los noventa, no se puede concluir que se logró mayor calidad ni equidad en la educación (CUENCA, 2014). De igual forma, hay que agregar que las reformas educativas implementadas en la mayoría de los países, aunque priorizaron la calidad educativa, también promovieron una descentralización que debilitó y fragmentó la estructura educativa (LAIES; DELICH, 2009). La preocupación por el poco avance posterior a muchas reformas es asumida por gobiernos, tomadores de decisiones y sociedad civil por la imposibilidad de alcanzar una educación inclusiva y de calidad. Pese a los esfuerzos de estos actores para alcanzar la inclusividad y la calidad educativa, las estrategias implementadas han tendido a enmendar - más que modificar - las estructuras organizativas centralizadas de los sistemas educativos latinoamericanos (PARDUCCI; VÁSQUEZ, 2019). Como parte de dichos esfuerzos, una de las propuestas más comunes tiene que ver con la reforma de las estructuras y los procesos de gobernanza, con miras a crear un estilo interdependiente entre el gobierno y las instituciones privadas y sociales; la coordinación más que la subordinación; con diálogo, negociación, colaboración y corresponsabilidad en la formación e implementación de políticas públicas (AGUILAR, 2010, p. 30-31). Como se hará claro, el presente ensayo toma como su enfoque la intersección de la descentralización y la participación en la gobernanza en el campo de la educación.

En la historia reciente de El Salvador, los cambios en la gobernanza han buscado la descentralización a través de cuatro estrategias: regionalización y nuclearización; gestión basada en la comunidad; departamentalización; y, actualmente, sistemas integrados. En cada uno, se identifican diferentes logros, duraciones e intereses por parte de actores nacionales y cooperantes internacionales; además, se observan diferentes grados de apropiación entre los actores locales y de la comunidad escolar, hacia la "buena gobernanza," o el arreglo que permite tener una "administración que rinda cuentas al público" y que empodera "a personas comunes [...] a tomar mayor responsabilidad para mejorar sus vidas" (WORLD BANK, 1989, p. xii; véase también EDWARDS Jr., 2014). No obstante, a través de los abordajes mencionados, estudios previos en El Salvador identificaron consistentemente cinco conclusiones desalentadoras. Las estrategias de descentralización: 1) carecen de claridad cuando se trata de dividir la autoridad; 2) se comunican de forma deficiente a todas las partes interesadas; 3 ) no se caracterizan por la inversión de los fondos y los recursos necesarios para su plena aplicación; 4) se ven obstaculizadas por marcos jurídicos y normativos inadecuados; y 5) 
reflejan una renuencia de las autoridades superiores a ceder el control (EDWARDS Jr., 2020).

Por estas razones proponemos que la participación más bien se conciba como una isla, apartada y aislada, en el mar de la descentralización. Es decir, cuando las comunidades deben contribuir en la gobernanza a través de su participación a nivel local, su razón de ser termina en la deriva y bajo un estado de abandono por la falta de apoyo necesario ( $v$ g., financiero, administrativo, legal, etc.), provocando que estas floten sin dirección en el océano de la reforma más amplia de la cual forman parte. Dicho de otro modo, aunque los actores locales (maestros, padres, directores de escuela, etc.) se esfuerzan por contribuir a la descentralización, su participación se caracteriza metafóricamente como una isla; esta se posiciona lejos de la tierra firme del apoyo gubernamental y dentro de un mar de reformas descentralizadoras más amplias que en sus diseños acarrean variadas formas de colaboración entre los diferentes niveles de la burocracia del Ministerio de Educación (MINED). Como se verá, el caso investigado representa muy bien lo planteado.

El presente ensayo considera estos resultados para estudiar la vigente estrategia de descentralización educativa implementada en El Salvador desde el 2011 conocida como: Sistema Integrado de Escuela Inclusiva de Tiempo Pleno (SI-EITP). El SI-EITP busca operar entre el nivel micro-político de la escuela y la organización sub-nacional a fin de mejorar la participación, la inclusión y la calidad. Bajo un análisis sistémico de la estrategia, se dará a conocer sus vaivenes en la construcción e implementación del modelo, sus logros y retos respecto a la descentralización y participación y la interrelación entre los diversos factores técnicos, institucionales y políticos que limitan su permanencia. En ese sentido, el ensayo tiene como objetivo explicar, por medio de un análisis sistémico, el funcionamiento del modelo SI-EITP en la práctica y las razones por las cuales opera de esa manera, así como en mostrar las formas de participación ciudadana que dicho modelo ha estimulado en la comunidad educativa inmersa en las dinámicas del SI-EITP. El argumento principal es que la descentralización en El Salvador aún refleja la carencia de soportes necesarios, de tal manera que los actores locales hacen lo mejor que pueden en un contexto donde el MINED no proporciona ni equipa las estructuras, los procesos y los recursos humanos mínimos para asegurar que la reforma se desarrolle tal como fue concebida. Si bien la intención es ofrecer una discusión de lecciones y recomendaciones que responden a la naturaleza del modelo de descentralización que representa SI-EITP, también el último apartado proporciona un segundo argumento que resulta del análisis: es necesario repensar la descentralización a nivel comunitario. Este último argumento recobra importancia, sobre todo si la meta es empoderar a las comunidades participantes para que puedan ejercer un papel que vaya más allá de un mecanismo técnico para ejecutar la gestión administrativa a nivel local. Como se enfatizará, este argumento se deriva no solo de la experiencia del SI-EITP, sino también de cuatro décadas de incumplimiento de las metas establecidas para las reformas de descentralización que aspiraron a generar participación a nivel local en El Salvador (EDWARDS Jr., 2020).

El ensayo partirá con una breve discusión de conceptos relevantes, luego los siguientes apartados abordarán el marco conceptual, los métodos de estudio y las características del modelo SI-EITP; posteriormente, se presentará una descripción de los hallazgos encontrados bajo este modelo; y para finalizar, se cerrará con una discusión, concluyendo con los retos - tanto técnicos, institucionales y políticos - que la implementación del modelo SI-EITP enfrenta en la práctica y con una reflexión sobre la necesidad de repensar la descentralización a nivel comunitario y su aporte a la participación en la educación.

\section{Gobernanza, Descentralización, Participación y Redes Escolares}

En este apartado, se hará una breve delimitación de los conceptos claves que sustentan el análisis, como gobernanza, descentralización, participación y redes escolares, los cuales son fundamentales para comprender la lógica detrás del modelo a estudiar. 
La gobernanza educativa, vista más allá de lo propuesto por Aguilar (2010), se delimita, frente a otros ámbitos, por cuatro factores principales: "el grado de descentralización del sistema en su conjunto; el tipo de financiamiento público-privado; los mecanismos de rendición de cuentas que se establecen y los actores relevantes que participan en la definición de la política educativa, sus intereses e incentivos" (VAILLANT, 2012, p. 120). El modelo de gobernanza, visto tanto a nivel macro (sistema educativo) como micro (escuela), tiene implicaciones en cuanto a actores, normativas, relaciones políticas y niveles de toma de decisiones, que son cuatro dimensiones que aportan al análisis de cómo se manifiesta la gobernanza en un sitio específico.

Además, la descentralización hace referencia a la transferencia de competencias o responsabilidades por parte del gobierno central a instituciones u órganos secundarios. Las competencias transferidas pueden incluir el planeamiento estratégico, la gestión de servicios, la recaudación de fondos, la asignación o distribución en el presupuesto, etc. (RONDINELLI; NELLIS; CHEEMA, 1983). Por consiguiente, existen diferentes grados de descentralización: desconcentración, delegación y devolución. Cada grado depende del nivel de poder que el gobierno central traslade a los niveles subordinados. Por ejemplo:

1. Desconcentración implica, generalmente, la transferencia de competencias - pero no de autoridad - a unidades dentro de una organización;

2. Delegación implica la transferencia de autoridad para la toma de decisiones por parte de las unidades superiores a las inferiores. Sin embargo, dicha autoridad puede ser revocada a discreción por la unidad superior;

3. Devolución se refiere a la transferencia de autoridad a una unidad autónoma que puede funcionar de forma independiente, o que puede actuar sin solicitar permiso alguno (HANSON, 1998, p. 112).

Como se verá, los Sistemas Integrados en El Salvador se colocan en un cruce entre la delegación y la devolución.

El impulso destinado a generar una participación en la educación se puede trazar desde la década de 1980, motivado, especialmente, por organismos internacionales tales como la UNESCO, el Banco Mundial y la USAID. Desde entonces, estos organismos han expresado distintas intenciones con relación a la participación. Una intención, asociada con la UNESCO, se ha orientado hacia el desarrollo social, la relevancia, la autonomía y la transformación, mientras que otra, asociada con el Banco Mundial, ha hecho más énfasis en la efectividad, la competitividad, la rendición de cuentas y el desempeño (EDWARDS Jr., 2014). Por su parte, la USAID se ha enfocado en la habilidad de la descentralización para contribuir al desarrollo democrático de los países de la región (EDWARDS Jr., 2018).

Una de las manifestaciones organizativas que ha estimulado la participación en educación es la conformación de redes, es decir, la agrupación de escuelas pertenecientes a una zona geográfica común bajo una sola unidad organizacional. La formación de escuelas en dichas agrupaciones puede ocurrir bajo la etiqueta de núcleos, clusters, redes o sistemas integrados. En estos, cada modelo puede responder a distintos propósitos, según las características de su diseño. Los propósitos pueden ser desde administrativos, pedagógicos o una combinación de ambos. Dado el involucramiento de los miembros de la comunidad en las tareas de gestión a nivel de la escuela, el modelo organizativo mencionado entremezcla la descentralización con la participación local, con el fin de alcanzar objetivos diversos como la ampliación de cobertura; un mejor costo-beneficio y mayor eficiencia; gestión descentralizada; y una mayor participación local basada en la autonomía y la rendición de cuentas (GIORDANO, 2008).

En El Salvador, se han desarrollado diversas experiencias con relación a las redes escolares. En la década de 1970 se organizó a las escuelas bajo “circuitos escolares", en los 80 en "núcleos", mientras que en los 
90 se les conoció como "escuelas modelo" orientadas principalmente a la mejora pedagógica (EDWARDS Jr., 2020). Posteriormente, en la zona rural y durante el Plan 2021 (2004-2009) se establecieron Redes Escolares Efectivas. Tales redes se integraron con las "redes solidarias" que se desarrollaron en los 100 municipios más pobres del país (MINED, 2005). Aunque el programa fue cesado al finalizar el periodo de gobierno, sirvió como experiencia fundacional para la formación del actual modelo de SI-EITP (FUSADES, 2019). Para facilitar el análisis del modelo actual, el siguiente apartado abordará tanto el marco conceptual como los métodos de investigación.

\section{Marco Analítico}

Como las reformas de gobernanza son, en su naturaleza, reformas al sistema de educación, es decir, reformas a las estructuras y procesos de gestión que caracterizan al sistema, es ventajoso - si no esencial - usar un marco conceptual que facilite un análisis del nuevo modelo de gobernanza que visualice las varias dimensiones del sistema. Así se podrá comprender la manera en que la interacción de diferentes dimensiones afecta el funcionamiento del nuevo modelo en la práctica. A tal fin, este estudio se basó en el marco de Gillies (2010). Este marco sistémico tiene tres dimensiones fundamentales e interactivas, a saber, la técnica, la institucional y la política. Brevemente, la dimensión técnica en el contexto educativo se refiere a los "elementos nucleares" -es decir, a los procesos, mecanismos, acciones, arreglos, etc .- que se encuentran en la base de una reforma educativa y que indican lo que ésta debería hacer en la realidad, o cómo debería funcionar la reforma en la práctica. En una visión sistémica, lo ideal sería que la dimensión institucional y política del sistema educativo apoyaran el cumplimiento de los aspectos técnicos en la práctica.

La dimensión institucional de un sistema educativo establece los parámetros mediante los cuales será posible que los lineamientos técnicos puedan operar. En términos analíticos, la dimensión institucional se compone de tres consideraciones: 1) el marco institucional del sistema, o más bien, "las políticas, procedimientos, normas, incentivos y modelos mentales" que apoyen o desestimen la reforma (GILLIES, 2010, p. 38); 2) la capacidad institucional o la "experticia de las organizaciones" que conforman el conjunto; y 3) los recursos disponibles en términos humanos, financieros y materiales. La interacción de estos tres aspectos de la dimensión institucional en un sistema educativo "crea los incentivos (o desincentivos) que harán efectiva la implementación de las soluciones técnicas" (GILLIES, 2010, p. 37-38).

Por último, la dimensión política subyace y permea la implementación de la reforma al sistema educativo. La cuestión fundamental de esta dimensión es quién o cuáles actores sostienen intereses en que la reforma educativa tenga éxito o fracase; $y$, qué papel desempeñan dichos actores al momento de fomentar u obstaculizar su implementación.

Para este caso, el marco analítico descrito ofrece la posibilidad de examinar los detalles sin perder de vista el sistema en su conjunto. En otras palabras, la visión sistémica permite analizar específicamente una serie de factores técnicos, institucionales y políticos determinantes, al tiempo que permite ver generalmente la identificación de "las fuerzas que interfieren en el proceso y que podrían llegar a sacudir o a impulsar una sociedad y, con ello, a facilitar o a impedir el cambio (GILLIES, 2010, p. 39). Aclarado esto, se desarrollará en la siguiente sección los métodos de investigación.

\section{Métodos de Investigación}

Es intrigante cómo el modelo SI-EITP ha recibido mucho interés en términos de financiamiento y atención durante varios años, pero muy poco de naturaleza académica o científica como para elaborar una sistematización de publicaciones sobre el tema. Para llenar esta brecha, se realizó una revisión exhaustiva 
de la documentación existente sobre SI-EITP, incluyendo tesis de maestría, documentos de conferencias no publicados, estudios de caso, así como informes y documentos internos, de trabajo y evaluaciones institucionales. Las referencias completas para la literatura analizada se encuentran en Edwards Jr. (2020), un estudio más amplio que se enfoca en los retos de la descentralización en El Salvador durante cuatro décadas.

En total, se encontraron y se revisaron 21 documentos, se identificaron conceptos clave y se ordenó la información relevante según la manera en que correspondía a cada dimensión del marco conceptual. Una vez agrupada, la información extraída fue sintetizada. Los puntos esenciales para comprender el caso de los SI-EITP se presentarán a continuación. La revisión detenida de la literatura también hizo posible construir una línea de tiempo sobre la formulación de la política, permitiendo la identificación de sus etapas de desarrollo y, más importante, comparar y contrastar los actores, los enfoques y los efectos en distintos momentos del desarrollo.

\section{El Modelo Sistema Integrado de Escuela Inclusiva de Tiempo Pleno (SI-EITP)}

Durante el periodo 2009-2019, el MINED implementó el Plan Social Educativo (PSE), el cual funcionó bajo tres principios básicos: establecer escuelas inclusivas que eliminen todas las barreras de acceso; la escuela como núcleo (centro) de cultura y la extensión del horario escolar con actividades curriculares ampliadas ( $v . g$., talleres, deporte, arte). Estos principios emergieron en un modelo de escuela denominado "Escuela Inclusiva de Tiempo Pleno" (EITP). El enfoque central de EITP es que todos los niños, niñas y adolescentes tengan el derecho de ir a la escuela y recibir una educación de calidad; para cumplir esa meta, fue necesario contar con recursos adicionales y con la participación de otros actores, principalmente locales.

En el 2009, el sistema educativo salvadoreño contaba con un total de 5,179 escuelas, del cual el 58\% de ellas eran consideradas pequeñas y disponían de cuatro o menos docentes para atender a los alumnos (MINED, 2014d). Pese a que el modelo EITP implicó atribuir responsabilidades adicionales a escuelas con limitados recursos, fue necesario pensar en la formación de redes escolares. En consecuencia, en el 2011 se denominó al modelo "Sistemas Integrados de Escuela Inclusiva de Tiempo Pleno" (SI-EITP). La formación de los Sistemas Integrados (SI) se ha materializado en la práctica a través de la creación de redes de hasta diez escuelas en estrecha proximidad geográfica, con el fin de facilitar el intercambio de recursos materiales y humanos y hacer cumplir la visión de EITP.

La noción de SI-EITP refleja la formación de redes para: 1) la ampliación de los servicios más allá de los temas generales del currículo; y 2) una mayor participación de los sectores locales en la vida de la escuela como una forma de procurar más recursos para la escuela, mejorar la estrategia de cohesión social, responder a las necesidades locales, fortalecer la gobernanza y mejorar la calidad. No obstante, no siempre está claro -dentro de cada nivel del sistema educativo - cómo se van a entrelazar en la práctica los diversos ideales de este modelo, como se presenta en el análisis en seguida.

\section{Dimensión Técnica}

Desde el punto de vista pedagógico, el modelo SI-EITP prevé una transformación de todas las actividades e iniciativas de la escuela, incluidos los procesos administrativos, a fin de fortalecer el aprendizaje. Cada escuela debe elaborar un proyecto pedagógico basado en sus necesidades de aprendizaje, y los grupos de escuelas organizadas en SI deben trabajar juntas en torno a un proyecto común. El currículo de las 
escuelas debe adaptarse a los diferentes contextos y capacidades del estudiante (WORLD BANK, 2011). Además, mediante una enseñanza organizada por equipos interdisciplinarios, las escuelas deben ayudar a los estudiantes a realizar proyectos que pongan en práctica los conocimientos adquiridos (USAID, 2010); asimismo, promueve el trabajo en equipo docente según el nivel y área de especialización para compartir experiencias, estrategias y recursos (BUITRAGO; SANTOS, 2016). Las diferentes partes de la estrategia están orientadas y apoyadas por los asistentes técnicos de las oficinas departamentales del MINED.

Desde el punto de vista organizativo, el modelo SI-EITP es posiblemente el más ambicioso y complejo de todas las iniciativas de descentralización en El Salvador hasta la fecha. A diferencia de otros enfoques de descentralización implementados, el MINED proporcionó directrices detalladas que especifican las estructuras a través de las cuales deben funcionar los SI-EITP (MINED, 2014a), con la creación de múltiples y nuevas instancias en distintos niveles de responsabilidad. Por ejemplo, a nivel de SI, se instala un Organismo Colegiado de Coordinación del Sistema Integrado (OCCSI), que gestiona todo el Sistema; luego, se establecen cuatro consejos de apoyo técnico y consultivo al director del OCCSI (Consejo Pedagógico, Consejo de Padres, Consejo de Directores y el Consejo Estudiantil). Adicionalmente, se conforman tres unidades de apoyo administrativo y curricular: 1) Coordinación de Áreas de Formación; 2) Coordinación de Niveles Educativos; y 3) la Instancia Colegiada de Administración (MINED, 2014a). Estas unidades coordinan de manera descendente con cada escuela y sus respectivas comisiones de trabajo; y ascendente con la Dirección Departamental de Educación (DDE) porque todo "depende jerárquicamente de la Dirección Departamental de Educación correspondiente" (MINED, 2014a, p. 30). Sin embargo, la naturaleza de la relación entre el organismo principal, el OCCSI, y las DDE no está del todo clara, como se explica en mayor detalle en lo que sigue.

Si bien el diseño de este modelo es a la vez ambicioso e intrincado, una cosa está clara: el nuevo modelo hace hincapié en un fuerte liderazgo educativo por parte del director de la escuela, el trabajo en equipo entre los docentes y la colaboración de los actores de la escuela con la comunidad local (WORLD BANK, 2011, p. 63). Las complejidades del modelo se expresan en su implementación, que se presentan a continuación.

\section{Implementación}

A pesar de la falta de claridad en el diseño, el modelo comenzó en 2011 con 22 escuelas, y luego se amplió a 38 en 2012, con la asistencia técnica y financiera de organismos internacionales (MINED, 2012). A mediados del 2013 se crearon 259 SI-EITP que beneficiaron a 2.082 escuelas (aproximadamente $40 \%$ del sistema público) en 92 localidades (MINED, 2015a). Los planes iniciales propusieron colocar a todas las escuelas en la modalidad SI-EITP para el año 2019 con una inversión de US\$ 98.47 millones del Banco Mundial junto con la Cooperación Italiana, USAID y fondos del gobierno salvadoreño, ${ }^{1}$ como evidencia de una estrategia de alta prioridad.

El MINED central y los socios internacionales trabajaron en los aspectos técnicos y financieros del modelo, mientras que el personal de las oficinas departamentales ayudó a expandir el modelo en todos los departamentos del país a través de “jornadas de sensibilización” (HERNÁNDEZ; RAMÍREZ; VENTURA, 2016, p. 25). Concretamente, los equipos de asistencia técnico-pedagógicos de las DDE trabajaron en los aspectos organizativos y de planificación en el terreno, como la agrupación de escuelas, la programación de actividades, y la estrategia del seguimiento y monitoreo (MINED, 2013). A nivel escolar, los estudios existentes destacan la participación de los estudiantes y las familias (MARTIN, 2017). Sin embargo, se ha encontrado una desconexión entre las expectativas y la realidad del modelo SI-EITP porque el concepto de participación 
se asoció a la simple asistencia y obligatoriedad de los involucrados a las actividades (MARTIN, 2017).

Ahora bien, no todos los estudios comparten este hallazgo. Mientras algunos padres de familia informaron que fueron convocados y reunidos solamente para asignarles responsabilidades en el marco del SI-EITP, otros mencionaron que su participación "incluye apoyo en excursiones, el mantenimiento de las escuelas, cuidar salones de clase en ausencia de los docentes, apoyo en los talleres, elaboración de periódicos murales y participar en proyectos financiados por organismos internacionales" (LUQUE, 2016, p. 116). Además, se descubrió que "los padres y madres de familia que no pertenecen a los organismos legítimos tienen participación” (LUQUE, 2016, p. 88). Los estudiantes, por su parte, mencionaron que su participación consiste en asistir a los talleres organizados por su SI local, mientras los docentes trabajaron en el proyecto pedagógico.

Aún con las descripciones proporcionadas no queda claro si la participación de la comunidad en el modelo SI-EITP refleja o no el tipo de cohesión social y solidaridad que el PSE expresó. A fin de cuentas, parece que las familias son simples receptores de información sobre las actividades escolares, sobre el funcionamiento del modelo educativo y sobre las decisiones elegidas por el MINED. Es más, no se observan mecanismos de retroalimentación sobre alguna falla presente en el modelo educativo.

En lo que respecta a los estudios que abordan el SI-EITP en la práctica, estos informan que los OCCSI, la unidad organizativa clave de supervisión en el conjunto de escuelas, no han funcionado como deberían. Por ejemplo, los miembros del OCCSI, que deberían ser siete (MINED, 2014a), percibieron que su participación no era significativa y que no entendían bien su función o papel dentro del modelo (LUQUE, 2016).

A la luz de lo anterior, no debe sorprender que los dos estudios cuantitativos del SI-EITP muestran resultados que, en el mejor de los casos, son mixtos en cuanto a los resultados positivos y negativos documentados (PINTO et al., 2014; SÁNCHEZ, 2018).

\section{Dimensión Institucional}

\section{Marco Legal y Directrices}

Al estudiar los conceptos sobre el SI-EITP, se encuentran desde sus inicios ciertas contradicciones en los propósitos y las explicaciones del "problema" educativo. Aunque la propuesta surgió a partir de las experiencias en inclusión educativa de la Cooperación Italiana, esta tomó fuerza bajo la lógica de la reorganización administrativa y territorial de USAID ante una necesidad urgente de maximizar recursos en escuelas rurales pequeñas (HERNÁNDEZ; RAMÍREZ; VENTURA, 2016). En contraste, el marco teóricofilosófico planteado en el PSE a partir del 2009 enfatizó la participación y la integración de la escuela a la comunidad en apoyo al derecho de la educación y la inclusión. En el 2011, un estudio de USAID planteó objetivos orientados a la eficiencia de los recursos (USAID; MINED, 2011), y para el 2013, se ampliaron los objetivos del modelo para mejorar la práctica pedagógica, garantizar el acceso a los servicios educativos, e impulsar un nuevo modelo de organización y administración escolar (MINED, 2013).

Con lo anterior se observa la convergencia de distintas perspectivas sobre las necesidades educativas -inclusión, recursos, participación comunitaria- en un modelo que dificultaría su implementación a raíz de los distintos objetivos expresados (USAID; MINED, 2011; LUQUE, 2016; HERNÁNDEZ; RAMÍREZ; VENTURA, 2016). Adicionalmente, es irónico que un modelo que promueve la participación terminó siendo una construcción vertical, producto de las decisiones tomadas desde el MINED central.

En esta línea, al consultar las visiones sobre la nueva organización en los SI-EITP, diversos directores de un mismo sistema expresaron formas variadas de comprender las intenciones educativas. Con respecto al 
OCCSI, Luque (2016) indica que los OCCSI no estaban convencidos del diseño del modelo y la participación de sus miembros dependía más de las preferencias y prioridades del director del SI (MARTIN, 2017). En el sector docente, se percibió mucha confusión sobre el PSE, evidencia de las carencias en la divulgación vertical de planes educativos desde el MINED central (LUQUE, 2016). La divergencia de conceptualizaciones sobre el modelo evidencia algunos retos institucionales que no han permitido avanzar en los objetivos esperados.

Adicional a los cambios repetidos en el diseño del modelo, la falta de una base legal ha creado desafíos adicionales. En el ámbito de la organización, se tuvo que hacer un viraje en el organismo rector de cada SI-EITP, pasando de ser el Organismo Directivo de Escuela Inclusiva (ODEI), a ser un Organismo Colegiado de Coordinación de Sistema Integrado (OCCSI), precisamente para hacer más expeditos los procesos de participación, con el agravante que el OCCSI no estaba legalizado (HERNÁNDEZ; RAMÍREZ; VENTURA, 2016).

Lo que complicó aún más la realidad del enfoque del SI-EITP fue que el marco institucional del propio MINED, el cual experimentó cambios significativos. Estos cambios se abordan en la siguiente sección.

\section{Capacidad y Recursos}

No sólo hubo cambios en el modelo SI-EITP, sino que también en las estructuras institucionales que debían apoyar la implementación de este modelo. En el 2009, el MINED central ofreció inicialmente apoyo a través de dos coordinaciones dependientes a la Dirección Nacional de Educación, una para dar seguimiento al EITP y la otra, a la estrategia de "nuclearización" o formación de redes. Sin embargo, en 2011, como parte del convenio del préstamo, el Banco Mundial indicó la creación de una instancia dedicada únicamente a la coordinación "día al día” del modelo SI-EITP, con el fin de mejorar la capacidad del MINED en desarrollar el modelo a nivel nacional. Esta instancia, denominada Dirección Adjunta de SI-EITP, fue liderada por un exministro de educación y velaba por todos los aspectos de implementación, con fuerte énfasis en los procesos financieros y de adquisiciones (WORLD BANK, 2011). La Dirección Adjunta estuvo en vigencia durante tres años hasta el 2015, luego fue disuelta por otra administración educativa y las actividades relacionadas con el SI-EITP fueron desarrolladas en el marco del proyecto Fomilenio o con apoyo de algunas direcciones departamentales de educación. Posteriormente, el enfoque del SI-EITP se integró transversalmente en la labor del MINED a través de la Unidad Coordinadora de Proyectos Estratégicos que fue creada en cada Dirección de Educación del MINED. No obstante, en el organigrama del MINED (2014-2019), no está contemplada dicha Unidad. El centro de responsabilidad institucional, además de pasar por varios cambios, fue absorbido y diluido en esta última etapa de gobierno.

Otro vacío en la dimensión institucional se refiere al financiamiento; el MINED no contaba con los recursos financieros necesarios para implantar el modelo y, por tanto, se vio supeditado a ejecutar el nuevo proyecto con préstamos y donaciones internacionales (BUITRAGO; SANTOS, 2016). En tal sentido, las fuentes de financiación vinieron acompañadas de diferentes enfoques sobre cómo implementar el modelo SI-EITP porque las prioridades de los donantes y la asistencia técnica proporcionada por cada uno es distinta (LUQUE, 2016). En todas las formas de apoyo, las estimaciones a partir de 2015 indicaban que se habían creado 190 Sistemas Integrados, que atendían a 417.333 estudiantes en 62 municipios (CRESPIN, 2015). El mayor financiador del modelo ha sido el Banco Mundial, cuyos recursos se utilizaron para financiar 101 de los 190 Sistemas Integrados. Para el 2024 se tenía proyectado cubrir los 262 municipios con un total de 689 Sistemas (CRESPIN, 2015), aunque, como se detalla arriba, los planes educativos desde el 2014 no contemplan este modelo.

Por supuesto, la capacidad institucional depende de algo más que recursos financieros. La asistencia técnica aportada por las organizaciones internacionales formaba parte del panorama. Si bien la consistencia en la asistencia técnica ofrecida por las diferentes organizaciones fue incierta existe cierta documentación 
que indica que, dentro del gobierno, no ha existido continuidad laboral del personal clave o estos han sido transferidos a otras oficinas dentro del MINED (BUITRAGO; SANTOS, 2016). Buitrago y Santos, basados en su estudio, escriben: "la investigación permitió identificar que los técnicos y diseñadores involucrados en los Sistemas Integrados de la EITP ya no ocupaban su cargo o habían sido transferidos a otras instancias del MINED (2016, p. 101).

Al descender del nivel central al nivel de la escuela, la mayor preocupación con respecto a la capacidad institucional de asumir el modelo radica en el tema de recursos, administración y coordinación entre escuelas (MARTIN, 2017). Es decir, el postulado de compartir recursos materiales y humanos, como computadoras, bibliotecas o, incluso, docentes entre escuelas de un mismo sistema requería un nivel de coordinación y confianza que no siempre existía. En algunas zonas del país, no había mucha convivencia ni trabajo en equipo entre escuelas, por lo que esto presentaba un nuevo reto. En otros casos, los directores manifestaron que ya estaban trabajando informalmente en redes, a raíz de las mismas necesidades sentidas. Surgían también cuestiones administrativas como la falta de nombramiento de directores y el doble papel de directores con un grado a su cargo (MARTIN, 2017).

También existían preocupaciones sobre el número y la capacidad de los docentes requeridos para apoyar adecuadamente el modelo del SI-EITP. En el 2009 se contabilizó que casi un 50\% de las escuelas contaron con una planta docente conformada por cuatro o menos docentes (MINED, 2014c), reflejando la poca capacidad de recursos humanos para desarrollar las estrategias del modelo. La etapa de formación docente en servicio se programó y ejecutó en una segunda administración educativa (2014-2019), pero se desvinculó del modelo SI-EITP, evidenciada en el Plan Nacional de Formación Docente que ni siquiera menciona la estrategia (MINED, 2015b). Sin embargo, una gran parte del reciente préstamo del Banco Mundial (para el proyecto, Mejoramiento de Calidad Educativa) se utilizó para la actualización docente, mas no para preparar al resto de actores que conforman el SI-EITP.

Como punto final de esta sección, se destaca que existió un síntoma institucional adicional y era la falta de incentivos que fomenten la coordinación interinstitucional. La razón de estos hallazgos, en parte, radicó en que la rendición de cuentas fluyó en la dirección equivocada, desde el gobierno hacia los socios internacionales, y no hacia las comunidades, escuelas y estudiantes atendidos (BUITRAGO; SANTOS, 2016).

\section{Dimensión Política}

Desde el punto de vista político, el apoyo al enfoque del SI-EITP ha sido inestable y errático. Por ejemplo, después de implementarse en pequeña escala y sin una evaluación de impacto formal, en el 2013 se inició una etapa de expansión masiva a 150 SI-EITP en 53 municipios, representando casi una tercera parte de las escuelas del país (MINED, 2013; HERNÁNDEZ; RAMÍREZ; VENTURA, 2016). Esto provocó un apresuramiento en la expansión, es decir, un conjunto de acciones aceleradas que no permitieron una implementación más sistemática y contextualizada. Políticamente, hubo un compromiso de alto nivel durante un periodo político, pero este nivel de compromiso duró solo tres años, como se ha descrito anteriormente, hasta que se transfiriera la responsabilidad del enfoque EITP transversalmente en el MINED. Aunque se necesita más información sobre cómo las DDE gestionan el enfoque EITP a través de las unidades estratégicas que se crearon en cada departamento, no es de esperar que las tareas relacionadas con el enfoque SI-EITP se hayan llevado a cabo en su totalidad, a menos que también se haya transferido la asistencia técnica de los donantes.

Por último, se debe tener en cuenta la situación de inseguridad que afecta a muchas comunidades en todo el país, las cuales se ven afectadas por un contexto de violencia pandilleril y social (USAID; ECCN, 
2016). El control territorial de las pandillas ha inhibido la organización de escuelas en Sistemas Integrados y afectado la posibilidad de compartir recursos entre escuelas por la peligrosidad de trasladar estudiantes de una zona a otra.

\section{Retos de la Descentralización Bajo el Modelo SI-EITP: Un Resumen con Recomendaciones}

Es posible subrayar que la experiencia del programa ha dado razón para optimismo cauteloso. En términos generales, como estrategia de descentralización y participación, se puede señalar que hubo:1) comunidades locales dispuestas a contribuir a la educación; 2) una implementación inicial de amplio alcance (190 Sistemas Integrados en 62 municipios); 3) una mayor participación en el diseño de la implementación de la estrategia a nivel de direcciones departamentales; y 4) el énfasis en recursos para el aprendizaje, inclusión y la mejora de la calidad educativa. En términos más específicos es posible señalar otros aspectos que se caracterizan por ser parcialmente exitosos. Estos incluyen 1) la creación de la base jurídica necesaria para la descentralización; 2) la transferencia de autoridad desde el nivel central a los niveles inferiores de la administración; 3) la provisión de capacitación para las nuevas funciones y responsabilidades; y 4) el desarrollo de estructuras, directrices, sistemas de información y procesos de comunicación necesarios. A pesar de los esfuerzos realizados, estos resultaron ser insuficientes o inadecuados para fortalecer y sostener un modelo tan complejo.

Las razones de las limitaciones del modelo se han abordado a profundidad en las secciones anteriores, por lo que aquí se ofrece un resumen de los puntos clave con comentarios adicionales. Los retos se resumen en cinco temas que emergen en estrecha vinculación al estudiar la descentralización de la educación a través del modelo SI-EITP: 1) marcos legales inadecuados; 2) falta de claridad en el diseño entorno a la reforma de la descentralización; 3) sistemas organizativos confusos, inadecuados e inapropiados; 4) insuficiente capacitación e inversión de recursos; y 5) dinámicas entre el MINED y las organizaciones internacionales.

\section{Marcos Legales Inadecuados}

En este caso, la reforma de la descentralización entró en conflicto con las leyes existentes. Por ejemplo, el OCCSI, institución fundamental del SI-EITP, comenzó a funcionar sin una base legal que legitimó su accionar, aspecto que generó conflicto con las responsabilidades ya establecidas por la normativa del MINED. Por tanto, futuras iniciativas de descentralización deben asegurar que la reforma prevista no entre en conflicto con las leyes existentes y anticiparse a los conflictos legales a fin de planificar las enmiendas necesarias que permitan la implementación de la reforma sin problemas.

\section{Falta de Claridad en torno al Diseño de la Descentralización}

Muchas reformas a la descentralización han sido diseñadas con un sentido superpuesto o poco claro respecto a la división en la autoridad y las responsabilidades. A veces esto se debe a la falta de comunicación por parte del gobierno central hacia las instituciones subnacionales y locales. En el SI-EITP, los actores involucrados no tuvieron claro el diseño del programa y sus responsabilidades dentro del mismo. Ante ello, las 
futuras reformas de descentralización deberán, desde el principio, desarrollar directrices claras que delimiten no sólo las responsabilidades de los actores clave sino también los procesos en los que deberán participar. Luego, estas directrices tendrán que ser ampliamente difundidas y socializadas para asegurar su familiaridad.

\section{Sistemas Organizativos Confusos, Inadecuados e Inapropiados}

Este problema hace referencia a la sobreposición de factores organizativos de carácter redundante e imprecisos, los cuales son el resultado de la falta de integración entre los múltiples sistemas y procesos que requiere la descentralización educativa. En el SI-EITP, no está claro si funcionó con éxito el complicado y multinivel sistema de comités en el nivel escolar y a nivel del OCCSI, especialmente en los casos de escuelas pequeñas con pocos docentes. Además, se reportó la falta de incentivos para la coordinación entre las distintas oficinas y direcciones del MINED y una serie de cambios en la forma en que se gestionó la reforma de los SI-EITP a nivel nacional. En fin, además de tener una estructura compleja y abrumadora a nivel local, la reforma del SI-EITP se vio aún más afectada porque las estructuras de liderazgo y apoyo a nivel central eran inestables.

Por consiguiente, las iniciativas de descentralización deben tratar de desarrollar o utilizar aquellos sistemas organizativos que proporcionen de manera consistente y coherente la supervisión, el seguimiento y el apoyo, a fin de que contribuyan a hacer realidad la visión de la reforma. Lograr este cálculo es un delicado equilibrio que debe tener en cuenta los procesos organizativos existentes, la capacidad organizativa disponible y los recursos financieros institucionales. Si bien es posible hacer cambios o correcciones una vez iniciado un programa, la falta de coherencia y estabilidad organizativa pone en peligro el éxito de la descentralización en la práctica.

\section{Insuficiente Capacitación e Inversión de Recursos}

La socialización y capacitación en la estrategia del SI-EITP fueron insuficientes y ejecutados de arriba hacia abajo. Esto fue evidente ya que los actores clave - particularmente a nivel local - informaron que no tenían claro sus funciones y responsabilidades. En futuras reformas de descentralización, el MINED deberá tener previsto estrategias concretas para involucrar, informar y capacitar sistemáticamente a los actores locales.

Además, la descentralización educativa ha tendido a incluir muy poco personal de apoyo a las escuelas, como administradores, psicólogos y trabajadores sociales. Tal afirmación es parte de una desafortunada realidad para muchas escuelas en El Salvador, las cuales carecen de recursos básicos para cumplir con el modelo pedagógico ambicioso, EITP, el cual requiere de escuelas bien equipadas. Al principio fue difícil proporcionar los insumos necesarios a las escuelas por el contexto de un MINED con limitados recursos financieros; no obstante, los socios internacionales contribuyeron a solventar esa necesidad.

Dado lo anterior, en el futuro, es importante asegurar que las reformas de la descentralización vayan acompañadas de los recursos necesarios, de lo contrario la reforma seguramente fracasará y todos los involucrados se verán frustrados. Al mismo tiempo, es necesario anticipar los problemas que puedan surgir en la práctica cuando se asuma que las escuelas compartirán recursos entre sí, porque las escuelas tienen pocos incentivos para colaborar de esta manera. Y aunque los padres de familia y los docentes a menudo están dispuestos a ofrecer su tiempo y energía para apoyar a su escuela, las reformas futuras deben tener cuidado de no asignar demasiado, aun cuando sea trabajo no remunerado, ya que los padres de familia y los docentes ya tienen muchas exigencias en su tiempo. 


\section{Dinámicas entre el MINED y las Organizaciones Internacionales}

La descentralización suele parecer una iniciativa que proviene de las organizaciones internacionales, más que del gobierno nacional. Esto es así porque el progreso, el financiamiento y la ayuda técnica a la reforma ha dependido del continuo apoyo de organizaciones internacionales como USAID, el Banco Mundial, entre otros. Trabajar con numerosos socios puede ser ventajoso porque el apoyo recibido ayuda a realizar cambios que de otra manera no serían posibles; pero a su vez, tal apoyo externo conlleva ciertos riesgos, ya que cada fuente de financiamiento puede tener una visión diferente de cómo diseñar y/o implementar una reforma determinada.

\section{Hacia Una Conclusión: Repensar La Descentralización A Nivel Comunitario}

Si el SI-EITP fuera implementado según la visión de sus diseñadores y promotores, podría ser un modelo beneficioso para la escuela en el sentido de que aumentaría la participación de la comunidad en la vida de la escuela y resultaría, entre otras cosas, en la elaboración de un proyecto pedagógico comunal con contenido curricular que reflejaría el contexto y las prioridades locales. Lo anterior permitiría ver tales reformas como un elemento esencial de un enfoque más amplio en la construcción y el empoderamiento de la comunidad. Sin embargo, la experiencia de una implementación así no ha sucedido ni en el contexto del programa SI-EITP ni en la experiencia de otros experimentos previos a nivel de la comunidad en El Salvador (EDWARDS Jr., 2020). Por lo tanto, se sugiere la necesidad de repensar la descentralización a nivel comunitario, sobre todo si la meta es empoderar las comunidades participantes para que puedan ejercer un papel que vaya más allá de un mecanismo técnico para ejecutar la gestión administrativa a nivel local.

A tal fin, aunque recientes trabajos han puesto de relieve el papel que los directores escolares pueden desempeñar en la movilización de los miembros de comunidades marginadas para contribuir con la calidad de la educación (DEMATTHEWS; EDWARDS Jr.; RINCONES, 2016; EDWARDS et al., 2020), se argumenta que también es necesario considerar el capital social de la comunidad de manera más amplia. En términos sociológicos, es necesario ir más allá de la simple noción de capital social como capacidad técnica de la comunidad. El capital social puede reconceptualizarse en términos de cohesión (bonding), puente (bridging) y vinculación (linking) de capital. Aquí, el capital de cohesión se refiere a las relaciones de confianza y cooperación entre los miembros, el capital puente se refiere a las relaciones de respeto y mutualidad entre personas que saben que no son iguales en algún sentido socio-demográfico y el capital de vinculación se refiere a las normas de respeto y redes de relaciones de confianza entre personas que están interactuando a través de gradientes de poder o autoridad explícitos, formales o institucionalizados en la sociedad (SZRETER; WOOLCOCK, 2004, p. 654-655).

El punto subyacente aquí es que, para que la descentralización educativa a nivel de la comunidad sea significativa, los reformadores deben cambiar la forma en que piensan acerca de la descentralización y el papel de las comunidades en ella. Por supuesto, pensar de esta manera va de la mano en cambiar la forma en que los stakeholders trabajan con las comunidades. Si bien se han realizado estudios que amplían el enfoque de la participación comunitaria hacia el empoderamiento de la comunidad (GITTELL; VIDAL, 1998; WARREN; MAPP, 2011), quedan por hacer cambios significativos en múltiples frentes, desde los conceptos empleados en la elaboración de políticas hasta las estrategias desplegadas en el trabajo con las comunidades. Así, la estrategia de descentralización a nivel de la comunidad puede ser considerada como un elemento más en los esfuerzos en pro del desarrollo general encaminados a la organización, la construcción y el empoderamiento de la comunidad.

El comentario anterior resulta no solo de la experiencia del SI-EITP sino también de cuatro décadas de frustraciones por parte de las comunidades en El Salvador (EDWARDS Jr., 2020). Un rasgo consistente en 
la descentralización de la educación ha sido que el gobierno hace grandes promesas acerca de los beneficios de la estrategia y de la participación de los miembros de la comunidad en sus procesos, sin pensar en los insumos y el apoyo que la escuela y la comunidad necesitan para realizar la visión comunicada. No se puede asumir que las escuelas y las comunidades involucradas dispongan de las habilidades y de los recursos necesarios para lograr la descentralización. Para reiterar el punto clave de este ensayo, aún si las comunidades dominaran el capital humano y económico para poner en práctica la reforma descentralizadora a nivel local, esta, por su parte, no provocaría su empoderamiento porque estas reformas han tendido a enfocarse más en los procesos administrativos en donde el estado central quiere trasladar su responsabilidad a niveles inferiores. Si se desea cambiar la dinámica de descentralización a manera que las comunidades puedan ir más allá de cumplir con procesos de gestión escolar a nivel local, organizarse de forma más democrática y ejercer una suerte de liderazgo para realizar metas que incluyen pero que, a su vez, sobrepasan asuntos escolares, hay que repensar tanto los modelos de descentralización a nivel de la comunidad como la manera en que el gobierno y los organismos internacionales apoyan el desarrollo comunitario. Sin duda, una comunidad que no posee el capital humano, social y económico suficiente no va a poder aprovechar la descentralización de la educación ni mucho menos va a poder ejercer su voluntad en los otros ámbitos a los cuales hay que atender (como el bienestar de las familias, la salud, etc.) para asegurar que la educación a nivel local se lleve a cabo en comunidades que puedan ser capaces de apoyarla. Estos rasgos son cada vez más importantes cuando se reflexiona sobre las dificultades que ha experimentado el gobierno central tanto para asegurar una educación de calidad como para entregar a las comunidades marginadas los servicios sociales y las oportunidades económicas que una democracia vigorosa debe de proveer.

\section{Contribuciones de los Autores}

Planteamiento del problema y contextualización; Edwards Jr. DB; Martin P. Metodología y análisis; Edwards Jr. DB; Erazo M; Martin P. Redacción; Edwards Jr. DB; Erazo M; Martin P.

\section{Notas}

1. El plan estratégico de educación para el período de gobierno 2014-2019, aunque incluyó la estrategia de EITP, ni siquiera mencionó el modelo de SI (MINED, 2014b) al igual que el reciente Plan Cuscatlán del periodo de 2019-2024. Solo el proyecto FOMILENIO II, de la Millenium Challenge Corporation (www. mcc.gov) está fortaleciendo 45 SI en la franja sur del país en el periodo de 2015 a 2020.

\section{Referencias}

AGUILAR, L. Gobernanza: el nuevo proceso de gobernar. Cuidad de México: Fundación Friedrich Naumann Para la Libertad, 2010.

BUITRAGO, R. B.; SANTOS, B. M. Estudio de caso sobre el diseño y la implementación de la estrategia de sistema integrado en la escuela inclusiva de tiempo pleno en El Salvador. 2016. 120 f. Tesis (Maestría en Política y Evaluación Educativa) - Facultad de Postgrados, Universidad Centroamericana "José Simeón Cañas”, Antiguo Cuscatlán, 2016. 
CRESPIN, E. Sistematización del modelo de Sistemas Integrados de Escuela Inclusiva de Tiempo Pleno. San Salvador: MINED, 2015.

COLLETTA, N. J.; PERKINS, G. Participation in education. Environment Department Working Papers n. 1. Washington: World Bank, 1995.

CUENCA, R. La educación en tiempos de desigualdades. Políticas sociales y reformas educativas en América Latina.In: CECCHINI, S. et al. (ed.). Educación y políticas sociales: sinergias para la inclusión. Buenos Aires: IIPE/UNESCO, 2014. p. 259-286.

DEMATTHEWS, D.; EDWARDS Jr., D. B.; RINCONES, R. Social justice leadership and community engagement: a successful case from Ciudad Juárez, México. Educational Administration Quarterly, Columbus, v. 52, p. 711-753, 2016. https://doi.org/10.1177/0013161X16664006

EDWARDS Jr., D. B. El concepto del Banco Mundial de la participación en el desarrollo y la gobernanza de la educación: un análisis de su acercamiento y resultados. Revista Latinoamericana de Estudios Educativos, Ciudad de México, v. XLIV, n. 1, p. 13-46, 2014. https://doi.org/10.48102/rlee.2014.44.1.253

EDWARDS Jr., D. B. The trajectory of global education policy: community-based management in El Salvador and the global reform agenda. New York: Palgrave MacMillan, 2018.

EDWARDS Jr., D. B. Descentralización de la educación en El Salvador: cuatro décadas de experiencia y retos. Informe preparado para Proyecto USAID Gobernabilidad Municipal. San Salvador: USAID, 2020.

EDWARDS Jr., D. B. et al. Community participation and empowerment in marginalized contexts: leveraging parental involvement, adult education, and community organizing through social justice leadership. Compare: A Journal of Comparative and International Education, London, 28 jan. 2020. https://doi.org/10.1080/03057925.2020.1717927

FUSADES [FUNDACIÓN SALVADOREÑA PARA EL DESARROLLO ECONÓMICO Y SOCIAL]. Hacia una regionalización efectiva de servicios educativos con calidad y equidad. Informe de Conyuntura Social 2018-2019. San Salvador: Fusades, 2019.

GIORDANO, E. A. School clusters and teacher resources centers. Paris: UNESCO, 2008.

GILLIES, J. The power of persistence: education system reform and aid effectiveness case studies in long-term education reform. Washington: USAID/Equip 2, 2010.

GITTELL, R.; VIDAL, A. Community organizing: building social capital as a development strategy. London: Sage, 1998.

HANSON, M. Strategies of educational decentralization: key questions and core issues. Journal of Educational Administration, Bradford, v. 36, n. 2, p. 111-128, 1998. https://doi. org/10.1108/09578239810204345

HERNÁNDEZ, J. R.; RAMÍREZ, H. A.; VENTURA, S. N. Comprensión y prácticas de participación de los actores educativos en el diseño e implementación de la política educativa. Un estudio de caso vertical de los sistemas integrados de El Salvador. 2016. 125 f. Tesis (Maestría en Política y Evaluación Educativa) - Facultad de Postgrados, Universidad Centroamericana “José Simeón Cañas”, Antiguo Cuscatlán, 2016. 
LAIES, G. F.; DELICH, A. Sistemas educativos y cohesión social: la reconstrucción de “lo común” en los Estados nacionales del siglo XXI. In: COX, C.; SCHWARTZMAN, S. (eds.). Políticas educativas y cohesión social en América Latina. Santiago de Chile: Uqbar Editores, 2009. p. 175-230.

LUQUE, E. La participación como un elemento clave para el fortalecimiento del sistema educativo. Caso de estudio SI-EITP Zaragoza en el período 2011-2015. 2016. 141 f. Tesis (Maestría en Política y Evaluación Educativa) - Facultad de Postgrados, Universidad Centroamericana “José Simeón Cañas”, Antiguo Cuscatlán, 2016.

MARTIN, P. Las redes escolares y la mejora de la participación en educación en el Sistema Integrado de Escuela Inclusiva de Tiempo Pleno de El Salvador. San Salvador:, Edición del Autor, 2017. Mimeografiado.

MINED [MINISTERIO DE EDUCACIÓN]. Redes escolares efectivas. San Salvador: MINED, 2005.

MINED \{MINISTERIO DE EDUCACIÓN]. Memoria de labores 2011-2012. San Salvador: MINED, 2012.

MINED [MINISTERIO DE EDUCACIÓN]. El Sistema integrado de EITP. Nuevo modelo educativo en construcción. Presentación en Power Point para uso interno, San Salvador, mayo 2013.

MINED [MINISTERIO DE EDUCACIÓN]. Lineamiento de funcionamiento de la estructura organizativa del Sistema Integrado de Escuela Inclusiva de Tiempo Pleno. San Salvador: MINED, 2014a.

MINED [MINISTERIO DE EDUCACIÓN]. Ejes estratégicos del Plan Nacional de Educación en Función de la Nación. San Salvador: MINED, 2014b.

MINED [MINISTERIO DE EDUCACIÓN]. Módulos varios de capacitación. San Salvador: MINED, 2014c. Mimeografiado.

MINED [MINISTERIO DE EDUCACIÓN]. Educación de El Salvador en Cifras. 2009-2014. San Salvador: MINED, 2014d.

MINED [MINISTERIO DE EDUCACIÓN]. Memoria de Labores 2014-2015. San Salvador: MINED, 2015a. MINED [MINISTERIO DE EDUCACIÓN]. Plan Nacional de Formación de Docentes en Servicio en el Sector Público. San Salvador: MINED, 2015b.

PARDUCCI, A.; VÁSQUEZ, M. O. Hacia un modelo de colaboración solidaria: reflexiones culturales desde la educación. In: PARDUCCI, A. et al. (eds.). Siglo XX. Cultura, violencia y territorios. San Salvador: Instituto Nacional de Formación Docente, 2019.

PINTO, M. C. et al. Evaluación del Sistema Integrado de Escuela Inclusiva de Tiempo Pleno implementado por el Ministerio de Educación de El Salvador. (Estudio en las comunidades educativas del municipio de Zaragoza del departamento de La Libertad. San Salvador: Universidad Tecnológica de El Salvador, 2014.

REIMERS, F. Tres paradojas educativas en América Latina. Sobre la necesidad de ideas públicas para impulsar las oportunidades educativas. Revista Iberoamericana de Educación, Ética y Formación Universitaria, Madrid, v. 29, p. 131-155, mayo/ago. 2002. https://doi.org/10.35362/rie290954

RONDINELLI, D.; NELLIS, J.; CHEEMA, G. Decentralization in developing countries: a review of recent experience. Washington: World Bank, 1983. 
SÁNCHEZ, M. Informe final: evaluación de impacto del proyecto "Mejoramiento de la Calidad de la Educación". BIRF 8110 SV. San Salvador: BIRF/MINED, 2018.

SZRETER, S.; WOOLCOCK, M. Health by association? Social capital, social theory, and the political economy of public health. International Journal of Epidemiology, Oxford, v. 33, n. 4, p. 650-667, 2004. https://doi.org/10.1093/ije/dyh013

USAID [UNITED STATES AGENCY FOR INTERNATIONAL DEVELOPMENT]. Propuesta de estrategia para la reorganización territorial para impulsar la Escuela Inclusiva de Tiempo Pleno. San Salvador: USAID/MINED, 2010.

USAID [UNITED STATES AGENCY FOR INTERNATIONAL DEVELOPMENT]; ECCN [EDUCATION IN CRISIS AND CONFLICT NETWORK]. Rapid Education and Risk Analysis El Salvador. Final report 2016. San Salvador: USAID/ECCN, 2016.

USAID [UNITED STATES AGENCY FOR INTERNATIONAL DEVELOPMENT]; MINED [MINISTERIO DE EDUCACIÓN]. Propuesta de estrategia para la reorganización territorial para impulsar la escuela inclusiva de tiempo pleno en los centros oficiales del país. Presentación en Power Point para uso interno, San Salvador, feb. 2011.

VAILLANT, D. La gobernanza educativa y los incentivos docentes: los casos de Chile y Uruguay. Revista Uruguaya de Ciencia Política, Montevideo, v. 21, n. 1, p. 119-141, 2012.

WARREN, M.; MAPP, K. A march on dry grass: community organizing as a catalyst for school reform. Oxford: Oxford University Press, 2011.

WHEELER, C. W.; CHUARATANAPHONG, J.; KUNARAK, P. Improving basic education through collaboration and co-operation: school clusters in Thailand. In: SHELDON, S. (ed.). Collaborating for educational change: the role of teachers, parents and the community in school improvement. Paris: UNESCO/IIEP, 1992. p. 39-88.

WORLD BANK. Sub-Saharan Africa: from crisis to sustainable development. Washington: World Bank, 1989.

WORLD BANK. Project appraisal document on a proposed loan in the amount of US\$60 million to the Republic of El Salvador for an education quality improvement project. Washington: World Bank, 2011.

\section{Sobre los Autores}

D. Brent Edwards Jr. es Ph.D. en Política Educativa Internacional (University of Maryland) y M.A. en Educación (University of Pennsylvania). Se desempeña como Associate Professor en la University of Hawai'i at Mānoa. Sus publicaciones abordan el estudio de los procesos de reforma educativa desde el enfoque de la economía política. Edwards ha estudiado en profundidad el programa "Educación con Participación de la Comunidad”, en El Salvador, analizando no solo sus orígenes y evolución, sino también el proceso por el cual el programa se ha convertido en un modelo global adoptado en otros países del mundo. Sus publicaciones recientes incluyen dos libros: Global Education Policy, Impact Evaluations, and Alternatives: The political Economy of Knowledge Production y The Trajectory of Global Education Policy: Community-based Management 
in El Salvador and the Global Reform Agenda (ambos con Palgrave Macmilan).

Mauricio Enrique Erazo Alfaro es Licenciado en Historia por la Universidad de El Salvador y maestrando en Política y Evaluación Educativa por la Universidad Centroamericana “José Simeón Cañas”.

Pauline Martin tiene formación docente, grado de maestría en Política y Evaluación Educativa y es directora de dicha maestría en la Universidad Centroamericana "José Simeón Cañas" de El Salvador, Centroamérica. Su experiencia profesional de más de 20 años incluye cargos de la coordinación de las carreras y prácticas docentes y coordinadora técnica de diversos proyectos educativos con UCA-MINED y más recientemente es coordinadora e investigadora principal del programa de investigación "Escuelas en Situaciones de Riesgo y Conflicto", con múltiples fuentes de financiamiento. Sus áreas de investigación son: análisis de política educativa; formación docente; la evaluación educativa; violencia y escuela; y género en educación. Ha publicado y participado en congresos sobre estos temas.

Recibido: 4 jun. 2020

Aceptado: 28 abr. 2021 